Simona M. E. Boito, MD² Jacqueline A. M. Laudy, MD, PhD

Piet C. Struijk

Theo Stijnen, PhD

Juriy W. Wladimiroff, MD, PhD

Index terms:

Abdomen, US, 856.128, 856.12981

Fetus, central nervous system, 856.871

Fetus, gastrointestinal tract, 856.871

Fetus, growth and development, 856.871

Fetus, US, 856.128, 856.12981

Published online before print 10.1148 /radiol.2233010656

Radiology 2002; 223:661-665

Abbreviations:

$\mathrm{CV}=$ coefficient of variation

$3 \mathrm{D}=$ three-dimensional

${ }^{1}$ From the Departments of Obstetrics and Gynaecology (S.M.E.B., J.A.M.L., P.C.S., J.W.W.) and Epidemiology and Biostatistics (T.S.), Erasmus University Medical Centre, University Hospital Rotterdam-Dijkzigt, Dr Molewaterplein 40, 3015 GD Rotterdam, the Netherlands. Received March 22, 2001; revision requested April 26; final revision received November 7; accepted December 5 . Address correspondence to J.W.W. (e-mail: breur@gyna.azr.nl).

Current address:

${ }^{2}$ Department of Maternal and Child Health, Biology and Genetics, University of Verona, Italy.

o RSNA, 2002
Author contributions:

Guarantor of integrity of entire study, J.W.W.; study concepts, S.M.E.B., J.W.W., P.C.S.; study design, T.S., J.A.M.L.; literature research, S.M.E.B.; clinical studies, S.M.E.B., J.A.M.L.; data acquisition, S.M.E.B., J.A.M.L.; data analysis/interpretation, S.M.E.B., P.C.S., J.A.M.L.; statistical analysis, S.M.E.B., T.S., P.C.S.; manuscript preparation, S.M.E.B., P.C.S., J.A.M.L., J.W.W.; manuscript definition of intellectual content and editing, J.W.W., T.S. manuscript revision/review, S.M.E.B. J.W.W.; manuscript final version approval, J.W.W.

\section{Three-dimensional US Assessment of Hepatic Volume, Head Circumference, and Abdominal Circumference in Healthy and Growth- restricted Fetuses ${ }^{1}$}

PURPOSE: To establish reproducibility and normal values for fetal hepatic volume and its significance in identification of fetal growth restriction relative to head and upper abdominal circumferences according to a cross-sectional study design.

MATERIALS AND METHODS: Pregnant women $(n=135)$ underwent ultrasonography. The coefficient of variation (CV) for hepatic volume scans obtained at 0 and 20 minutes and hepatic area tracings, performed twice for each scan, was determined ( $n=20$; range, 23-36 weeks). Normal data for hepatic volume and head and upper abdominal circumferences were obtained ( $n=85$; range, 20-36 weeks) and related to data from growth-restricted fetuses (birth weight $<$ P5 centile; $n=24$; range, 22-36 weeks).

RESULTS: CV was $2.9 \%$ for volume scans and $1.6 \%$ for area tracings. In 85 uncomplicated cases, mean fetal hepatic volume (P50 centile) was $9.7 \mathrm{~mL} \pm 4.4$ (SD) at 20 weeks and $96.4 \mathrm{~mL} \pm 8.2$ at 36 weeks of gestation. In 24 growthrestricted fetuses, hepatic volume, head circumference, and upper abdominal circumference expressed as percentages of the normal P50 centile were $45 \%, 90 \%$, and $82 \%$, respectively. Mean difference in hepatic volume between fetal growth restriction and normal fetal development, as expressed with the $z$ score, $-4.32 \pm$ 1.4 , was significantly different $(P<.05)$ from that for head circumference, $-3.04 \pm$ 1.3 , but not from that for upper abdominal circumference, $-4.7 \pm 1.2$. Fetal hepatic measurement was obtained in 109 pregnancies.

CONCLUSION: Acceptable reproducibility exists for hepatic volume determinations. In fetal growth restriction, reduction is more pronounced for hepatic volume than for head or upper abdominal circumference; hepatic volume is a better discriminator than head circumference but not upper abdominal circumference.

o RSNA, 2002

Measurement of fetal upper abdominal circumference is considered the mainstay of ultrasonographic (US) determination of fetal growth and estimation of fetal weight $(1,2)$. The fetal liver makes up most of the abdomen, as measured by means of abdominal circumference. Measurement of fetal hepatic volume to identify fetal growth restriction is of interest, since both the human and the rat have severely depleted hepatic glycogen stores associated with growth restriction (3). Due to fetal malnutrition, reduction in fetal hepatic weight is more pronounced than is reduction in brain weight because of the brain-sparing effect, which reflects redistribution of fetal blood flow during chronic fetal hypoxemia (4). Measurement of fetal hepatic volume may thus contribute to the early detection of fetal growth restriction. Authors of another study (5) dealing with measurements of fetal hepatic length in fetal growth restriction suggest that other intraabdominal 
organs, reduced amounts of fat, or elevation of the diaphragm because of poor pulmonary growth also contribute to reduced fetal upper abdominal circumference.

Recently, a more direct approach to measurement of fetal hepatic volume by using three-dimensional (3D) US was reported (6), including preliminary data on normal fetal hepatic volume from our center (Erasmus University Medical Centre, University Hospital Rotterdam-Dijkzigt, Rotterdam, the Netherlands) (7). To our knowledge, no data are available about 3D US measurement of fetal hepatic volume in fetal growth restriction. Therefore, the purpose of our study was to establish reproducibility and normal values for fetal hepatic volume and its importance in identification of fetal growth restriction relative to head and upper abdominal circumferences according to a cross-sectional study design.

\section{MATERIALS AND METHODS}

During the period of April 1997 to October 2000, 135 women with a singleton pregnancy from our outpatient unit (Erasmus University Medical Centre, University Hospital Rotterdam-Dijkzigt, Rotterdam, the Netherlands) consented to participate in the study after approval was granted by the institutional ethics review board. This group included 34 normal singleton pregnancies from a previous study (7).

Technically acceptable US hepatic recordings were obtained in 85 of 104 uncomplicated pregnancies and in 24 of 31 pregnancies associated with fetal growth restriction, which resulted in success rates of $82 \%$ and $77 \%$, respectively. A US hepatic recording qualified as acceptable if the entire contour of the liver could be manually outlined. Recording failures were mainly due to maternal obesity, fetal position, or excessive fetal movement. A hepatic volume calculation took approximately 15 minutes. Each woman underwent evaluation only once in the study.

An uncomplicated pregnancy constituted the delivery of a healthy neonate with a birth weight between the 5th and 95th centile for weight of gestation, according to the Kloosterman tables (8). Fetal growth restriction was represented by fetal upper abdominal circumference below the 5th centile (9), and a birth weight below the 5 th centile for weight of gestation was confirmed after delivery, according to the Kloosterman tables (8).
Pregnancy duration was determined from last menstruation and confirmed by means of US before 20 weeks of gestation. Gestation was at 20-36 weeks (median, 30 weeks) in uncomplicated pregnancies and 22-36 weeks (median, 29 weeks) in the subset of pregnancies with fetal growth restriction. Maternal age in all 109 pregnancies was 19-42 years (median, 29 years).

The $5-\mathrm{MHz}$ annular array transducer (VSW 3-5; Kretz Technik, Zipf, Austria) of the US machine (Combison 530-D; Kretz Technik) was used for volume scanning. Two examiners (S.M.E.B. and J.A.M.L.) obtained the data and hepatic volume measurements.

Organ volumes were determined by slicing through collected images and recording a truncated pyramidal volume. Depth, longitudinal, and transverse dimensions of this volume were adjustable, with an initial area of $6.6 \times 3.3 \mathrm{~cm}$, up to a maximum area of $28 \times 22 \mathrm{~cm}$ at a depth of $20 \mathrm{~cm}$. These dimensions provide a maximum scanned volume of 5.9 L. The maximum resolution in the longitudinal direction was 512 ultrasound lines for each of the maximum of 500 sections in the transverse, or sweep, direction.

In the present study, the opening angle of each plane was $70^{\circ}$, and the angle of the volume sample was limited to a maximum of $60^{\circ}$. The depth of the volume was restricted to $12-16 \mathrm{~cm}$, with a resolution of 210 sections per volume. After each US scan, the volume was presented in three perpendicular planes on the monitor and represented frontal, sagittal, and transverse cross sections of the fetus. Data were stored on hard disk (Iomega Jaz Drive; Iomega, Roy, Utah) for off-line analysis.

In a first step toward fetal hepatic volume determination, a frontal cross section of the liver anterior to the stomach was visualized. In a simultaneously demonstrated sagittal cross section, the outline of the liver was traced manually in approximately 10 sagittal sections between the most lateral left and right points of the diaphragm in the frontal plane. The liver was measured from its upper limit at the diaphragm to its lower limit at the distal rim. The system automatically kept track of the distances between the sections and calculated the total volume after each area measurement was completed.

In each instance, fetal hepatic volume recording was followed by measurement of fetal head and upper abdominal circumferences and femur length. Fetal weight was estimated from these biometric parameters by using the Hadlock formula (10).

\section{Reproducibility}

The first 20 uncomplicated singleton pregnancies examined by S.M.E.B. were enrolled in the reproducibility study. Pregnancy duration was at 23-36 weeks of gestation. Hepatic volume recordings were obtained at 0 and 20 minutes to determine the intraobserver variability as expressed by means of the coefficient of variation $(\mathrm{CV})$. To establish the $\mathrm{CV}$ for fetal hepatic volume tracings, the tracings were performed twice for each hepatic volume recording-the one obtained at 0 minutes and the one obtained at 20 minutes. Analysis of variance was used to calculate the variance and the SD within the separate categories of recordings and tracings. The CV was calculated by dividing the SD by the overall mean of the measurements.

\section{Statistics}

The gestational age-related reference centiles-P5, P50, and P95-were constructed by using the method described by Altman (11). According to this procedure, we modeled the relationships among hepatic volume, head circumference, upper abdominal circumference, and gestational age by means of a cubic polynomial. Either the linear or quadratic term was left out because of too low a tolerance (SPSS version 8.0 for Windows; SPSS, Chicago, Ill). The age-specific SD was obtained from linear regression of the absolute residuals on gestational age. Polynomial linear regression was used to calculate the relationship between hepatic volume and estimated fetal weight.

Data from hepatic volume, head circumference, and upper abdominal circumference for fetal growth restriction were expressed as ratios. Every observed value of the growth-restricted fetus was divided by the corresponding normal P50 value for gestational age. Abdominal circumference and head circumference ratios were raised to the power of three to estimate the abdominal and head volume reduction in growth-restricted fetuses. These values were compared with the 3D hepatic volume data for fetal growth restriction.

Mean difference in hepatic volume between fetuses with growth restriction and those with normal development was determined with the $z$ score, which was calculated as follows: $z$ score $=$ hepatic vol- 


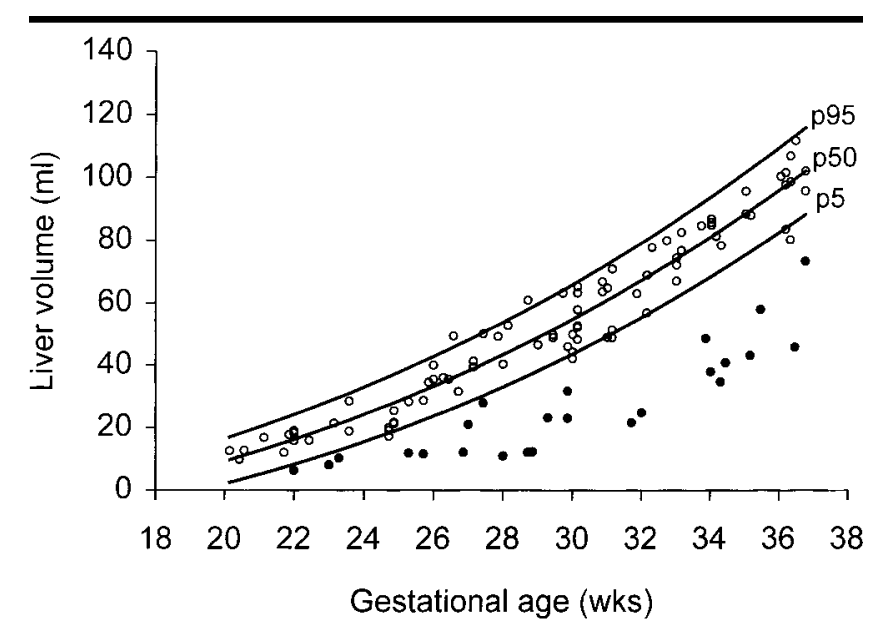

Figure 1. Graph shows hepatic volume in milliliters relative to gestational age in weeks. All hepatic volumes of the growth-restricted fetuses are below the P5 reference level. Note that these hepatic volume data are even more reduced relative to the P50 reference level than are the abdominal circumference data (Fig 3). However, the mean difference in hepatic volume between fetal growth restriction and normal fetal development ( $z$ score) is not significantly different from that for fetal upper abdominal circumference. $\mathrm{O}=$ individual normal values, $\bullet=$ individual fetal growth-restricted values. Solid lines represent the P5, P50, and P95 reference lines. For P50, cubic fit $=0.0012 \times \mathrm{GA}^{3}+0.0443 \times \mathrm{GA}^{2}-18.268$, where $G A$ is gestational age. P5 - P95 $=$ P50 $\pm 1.64(\mathrm{SD})(-0.2408 \times \mathrm{GA}-0.4560)$.

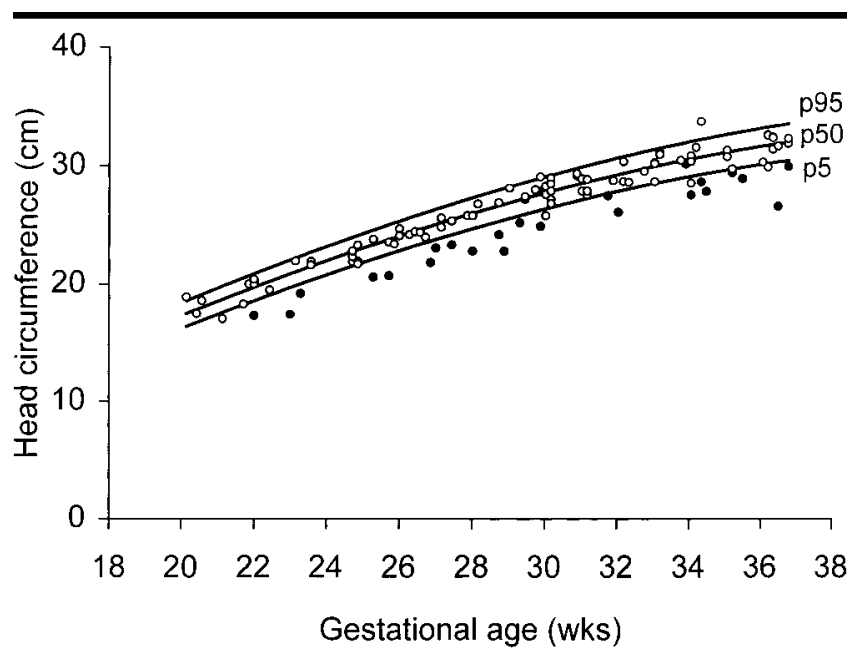

Figure 2. Graph shows head circumference in centimeters relative to gestational age in weeks. The head circumference data in the growth-restricted fetuses are less reduced relative to the P50 reference level than are hepatic volume and upper abdominal circumference data. However, the mean difference in hepatic volume between fetal growth restriction and normal fetal development ( $z$ score) is significantly $(P<.05)$ different from that for fetal head circumference. $\mathrm{O}=$ individual normal values, $\bullet=$ individual fetal growth-restricted values. Solid lines represent the P5, P50, and P95 reference lines. For P50, cubic fit $=-0.000274 \times \mathrm{GA}^{3}+1.564502 \times \mathrm{GA}-11.801200$, where $G A$ is gestational age. P5 $-\mathrm{P} 95=\mathrm{P} 50 \pm 1.64(0.016072 \times \mathrm{GA}+$ $0.343292)$ ume $_{\mathrm{FGR}}-\mathrm{P} 50_{\text {normal }} / \mathrm{SD}_{\text {normal }}$, where $F G R$ represents fetuses with growth restriction and "normal" represents fetuses with normal development. In the group with fetal growth restriction, the percentage of normal P50 values and $z$ scores of the hepatic volume were compared with upper abdominal and head circumference values by means of the paired sample $t$ test. The level of statistical significance was set at .05.

\section{RESULTS}

The number of confirmed normally developed fetuses was 85 and the number of growth-restricted fetuses was 24 . CVs for hepatic volume recordings and hepatic volume tracings were $2.9 \%(1.735 /$ $59.791)$ and $1.6 \%(0.968 / 59.791)$, respectively, which resulted in a total CV of $3.3 \%(1.986 / 59.791)$ for all fetuses. The number of uncomplicated pregnancies per gestational week varied between three and 12. Mean gestational age at delivery was 40.1 weeks \pm 1.2 (SD). In uncomplicated pregnancies, mean fetal hepatic volume was $9.7 \mathrm{~mL} \pm 4.4$ at 20 weeks and $96.4 \mathrm{~mL} \pm 8.2$ at 36 weeks of gestation (Fig 1). At 20 and 36 weeks, respectively, mean fetal head circumferences (Fig 2) were $17.5 \mathrm{~cm} \pm 0.7$ and 31.7 $\mathrm{cm} \pm 0.9$, and mean fetal upper abdom- inal circumferences (Fig 3) were 14.8 $\mathrm{cm} \pm 0.8$ and $31.4 \mathrm{~cm} \pm 1.0$ in uncomplicated pregnancies. A significant linear relation was established between fetal hepatic volume and estimated fetal weight $(P=.001)$ (Fig 4).

In fetuses with growth restriction, mean gestational age at delivery was 31 weeks \pm 4.6 , mean fetal birth weight was $1,140 \mathrm{~g} \pm 636$, and mean lag time between hepatic volume measurement and delivery was 2.5 weeks \pm 1.4 . Fetal hepatic volume, fetal head circumference, and upper abdominal circumference were below the 5 th centile of the reference chart in most or all cases (Figs 1-3).

Mean difference in hepatic volume between fetuses with growth restriction and those with normal development as expressed by means of percentage of the normal P50 values was $45 \% \pm 13.0$, which is significantly different $(P<.001)$ from head circumference, $90 \% \pm 4.2$, and fetal upper abdominal circumference, $82 \% \pm 4.1$. Within the subset of growth-restricted fetuses, mean hepatic volume was also significantly smaller $(P<.001)$ than the estimated head volume of $75 \% \pm 11$ and estimated abdominal volume of $56 \% \pm 8.4$. Mean difference in hepatic volume between fetuses with growth restriction and those with normal development, as expressed with the $z$ score, was $-4.32 \pm 1.4$, which is significantly different $(P<.05)$ from the $Z$ score for head circumference, $-3.04 \pm$ 1.3 , but not that for fetal upper abdominal circumference, $-4.7 \pm 1.2$.

\section{DISCUSSION}

The present study focused on 3D US measurements of fetal hepatic volume in normal and abnormal fetal development during the second half of pregnancy. The success rate in acquiring technically acceptable fetal hepatic volume measurements was as high as $82 \%$ in uncomplicated pregnancies and lower (77\%) in fetuses with growth restriction.

Low image quality occurred in the presence of maternal obesity or when the fetal back was in the anterior position. In the latter case, fetal bones, particularly vertebrae and ribs, caused shadows on some parts of the fetal abdomen, which limited the definition of some sections inside the volume box. Reduced amniotic fluid volume constituted a reason for not obtaining a technically acceptable hepatic volume measurement in the growthrestricted fetus. In the present study, the second half of pregnancy was not selected because of 3D hepatic volume measurement limitation before 20 weeks of gesta- 


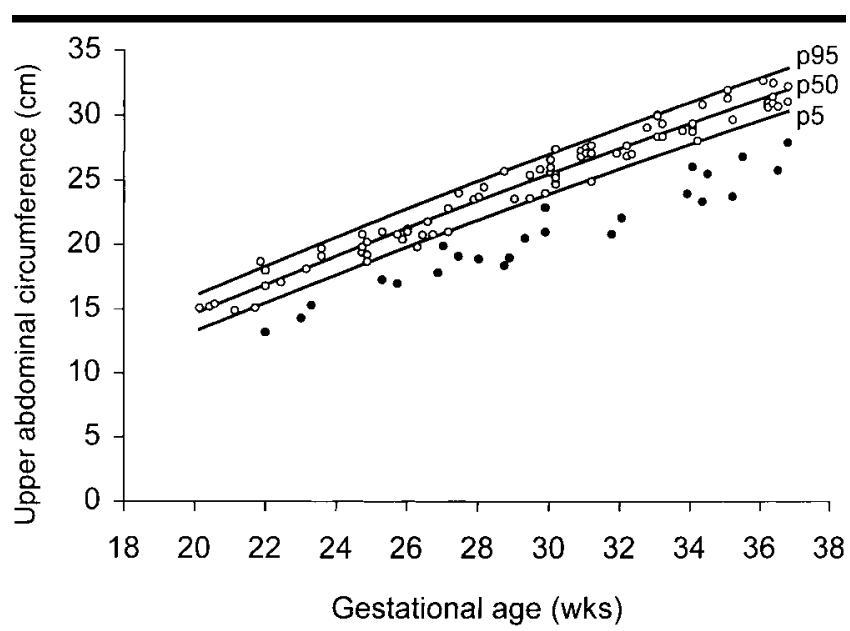

Figure 3. Graph shows upper abdominal circumference in centimeters relative to gestational age in weeks. This figure should be interpreted as a reference figure to be compared with Figures 1 and $2.0=$ individual normal values, $\bullet=$ individual fetal growth-restricted values. Solid lines represent the P5, P50, and P95 reference lines. For P50, cubic fit $=-0.000079 \times \mathrm{GA}^{3}+1.2381 \mathrm{GA}-9.493536$, where $G A$ is gestational age. $\mathrm{P} 5-\mathrm{P} 95=\mathrm{P} 50 \pm 1.64(0.009144 \times \mathrm{GA}+0.665685)$.

tion (6) but because fetal growth restriction usually does not begin until 22-24 weeks of gestation.

Acceptable reproducibility of hepatic volume measurements is essential before embarking on a clinical study. We demonstrated that the reproducibility of fetal hepatic volume recordings and tracings is good, with a total CV less than $4 \%$. We did not study the accuracy of fetal hepatic volume measurements with 3D US, but other investigations (12-14) have been performed to confirm the accuracy of 3D US volume measurements.

In uncomplicated pregnancies, fetal hepatic volume demonstrates a tenfold increase with advancing gestational age and increasing fetal weight. The regression line, shown in Figure 4, demonstrates that the hepatic volume is proportional to estimated fetal weight during the second half of pregnancy.

Currently, US measurement of fetal head and upper abdominal circumferences is the most widely used method of establishing impaired fetal growth $(15,16)$. The detection of fetal growth restriction by means of head circumference measurements may be limited because of fetal brain sparing in the presence of chronic fetal hypoxemia. Results of a majority of studies $(15,16)$ have shown that the most effective method of detecting fetal growth restriction is measurement of the upper abdominal circumference. However, this measurement is not satisfactory because the positive predictive value for detecting fetal growth restriction may be as low as

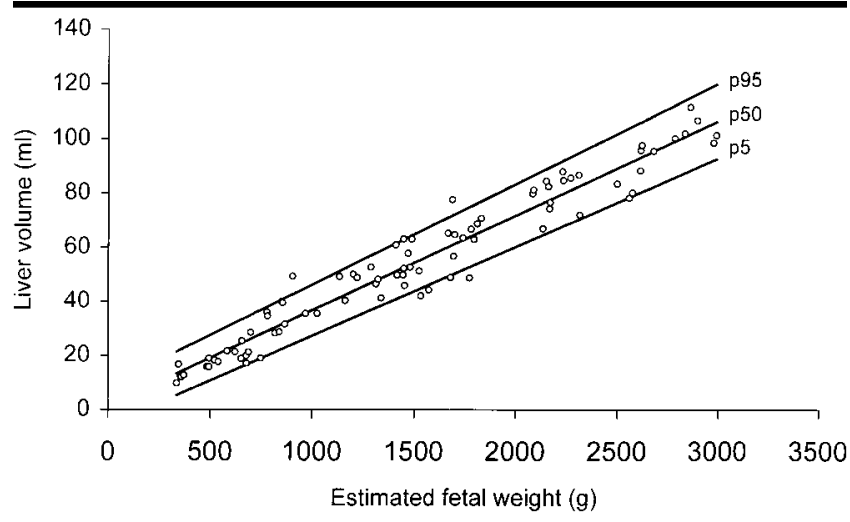

Figure 4. Graph shows hepatic volume in milliliters relative to estimated fetal weight in grams. P50 regression line demonstrates that hepatic volume is proportional to estimated fetal weight during the second half of pregnancy. $\mathrm{O}=$ individual normal values. Solid lines represent the P5, P50, and P95 reference lines. For P50, linear fit $=35.190623 \times \mathrm{EFW}+1.560381$, where $E F W$ is estimated fetal weight. P5 - P95 = P50 × $1.64(1.300713 \times \mathrm{EFW}+4.447085)$.

pressed with the $z$ score, we found a significant difference only when comparing these values with head circumference, which confirms the brain-sparing effect during abnormal fetal development.

Three-dimensional US measurements of fetal hepatic volume show good reproducibility. Normal fetal hepatic volume is tenfold larger at the end of gestation, as compared with that at the beginning of the second half of pregnancy. Fetal growth restriction affects fetal size, but fetal hepatic volume measurement is not a better discriminator for growth restriction than is upper abdominal circumference measurement. two-dimensional fetal hepatic length and fetal upper abdominal circumference in uncomplicated pregnancies $(18,19)$. Threedimensional US measurements of the normal hepatic volume demonstrated a close linear relation between hepatic volume and estimated fetal weight $(6,7)$. Baker et al (17) were able to show that fetal hepatic volume measurements obtained with magnetic resonance (MR) imaging allowed accurate detection of fetuses that were subsequently found to be growth restricted. However, MR imaging is associated with reduced patient acceptability and high cost.

The present study results show that fetal growth restriction is associated with reduced hepatic volume in every instance. Moreover, when expressed as a percentage of the normal P50, the decrease in hepatic volume is more pronounced than is the reduction in head circumference or upper abdominal circumference. When looking at the mean difference in hepatic volume between normal and reduced fetal growth, as ex-

\section{References}

1. Campbell S. The assessment of fetal development by diagnostic ultrasound. Clin Perinatol 1974; 1:507-524.

2. Campbell S, Wilkin D. Ultrasonic measurement of fetal abdomen circumference in the estimation of fetalweight. $\mathrm{Br} \mathrm{J} \mathrm{Ob-}$ stet Gynaecol 1975; 82:689-697.

3. Evans M, Mukherjee A, Schulman J. Animal models of intrauterine growth retardation. Obstet Gynecol Surv 1983; 38:183192.

4. Groenenberg IA, Wladimiroff JW, Hop WC. Fetal cardiac and peripheral arterial flow velocity waveforms in intrauterine growth retardation. Circulation 1989; 80: 1711-1717.

5. Roberts AB, Mitchell JM, McCowan LM, Barker S. Ultrasonographic measurement of liver length in the small-for-gestational-age fetus. Am J Obstet Gynecol 1999. 180:634-638

6. Chang FM, Hsu KF, Ko HC, Yao BL, Chang $\mathrm{CH}$, Chen HY. Three-dimensional ultrasound assessment of fetal liver volume in normal pregnancy: a comparison of reproducibility with two-dimensional ultrasound and a search for a volume con- 
stant. Ultrasound Med Biol 1997; 23:381389.

7. Laudy JAM, Janssen MMM, Struijk PC, Stijnen T, Wallenburg HCS, Wladimiroff JW. Fetal liver volume measurement by three-dimensional ultrasonography: a preliminary study. Ultrasound Obstet Gynecol 1998; 12:93-96.

8. Kloosterman G. On intrauterine growth. Int J Obstet Gynaecol 1970; 8:895-912.

9. Snijders RJM, Nicolaides KH. Fetal biometry at 14-40 weeks' gestation. Ultrasound Obstet Gynecol 1994; 4:34-38.

10. Hadlock FP, Harrist RB, Sharman RS, Deter RL, Park SK. Estimation of fetal weight with the use of head, body, and femur measurements: a prospective study. Am J Obstet Gynecol 1985; 151:333-337.

11. Altman DG. Construction of age-related reference centiles using absolute residuals. Stat Med 1993; 12:917-924.

12. Gilja OH, Thune N, Matre K, Hausken T, Odegaard S, Berstad A. In vitro evaluation of three-dimensional ultrasonography in volume estimation of abdominal organs Ultrasound Med Biol 1994; 20:157-165.

13. Brunner M, Obruca A, Bauer P, Feichtinger W. Clinical application of volume estimation based on three-dimensiona ultrasonography. Ultrasound Obstet Gynecol 1995; 6:358-361.

14. Riccabona M, Nelson TR, Pretorius DH Davidson TE. Distance and volume measurement using three-dimensional ultrasonography. J Ultrasound Med 1995; 14 881-886.

15. Seeds JW. Impaired fetal growth: ultrasonic evaluation and clinical management. Obstet Gynecol 1984; 64:577-584.
16. Warsof SL, Cooper DJ, Little D, Campbell S. Routine ultrasound screening for antenatal detection of intrauterine growth retardation. Obstet Gynecol 1986; 67:3339.

17. Baker PN, Johnson IR, Gowland PA, et al. Measurement of fetal liver, brain and placental volumes with echo-planar magnetic resonance imaging. $\mathrm{Br} \mathrm{J}$ Obstet Gynaecol 1995; 102:35-39.

18. Vintzileos A, Neckles S, Campbell WA, Andreoli JW, Kaplan BM, Nochimson DJ. Fetal liver ultrasound measurements during normal pregnancy. Obstet Gynecol 1985; 66:477-480.

19. Murao F. Measurements of the fetal liver size, hormonal level and pregnancy outcome. Gynecol Obstet Invest 1991; 32: 153-156. 